\title{
Does neighborhood disorder predict recovery from mobility limitation? Findings from the Health and Retirement Study
}

\author{
Kenzie Latham, Ph.D. ${ }^{1}$ \& Monica M. Williams, B.A ${ }^{1}$ \\ ${ }^{1}$ Department of Sociology, Indiana University-Purdue University Indianapolis, Indianapolis, IN \\ Corresponding Author: \\ Kenzie Latham, Ph.D. \\ Assistant Professor \\ Department of Sociology \\ Indiana University-Purdue University Indianapolis \\ Cavanaugh Hall-303E \\ Indianapolis, IN 46202 \\ 317-278-5204 \\ keelatha@iupui.edu
}

\section{RUNNING HEADER: NEIGHBORHOOD DISORDER \& RECOVERY}

Word Count: 6,568

Tables: 4

Figures: 1

Keywords: neighborhood disorder; mobility limitation; recovery; disability; aging; Health and Retirement Study

This is the author's manuscript of the article published in final edited form as:

Latham, K., \& Williams, M. M. (2015). Does Neighborhood Disorder Predict Recovery From Mobility Limitation? Findings From the Health and Retirement Study. Journal of aging and health, 0898264315584328. http://dx.doi.org/10.1177/0898264315584328 


\title{
Does neighborhood disorder predict recovery from mobility limitation? Findings from the Health and Retirement Study
}

\begin{abstract}
Objectives: This research explores whether perceptions of physical neighborhood disorder predict recovery from mobility limitation over a two-year period and examines whether psychosocial factors (i.e., depressive symptomology and mastery) and physical activity are salient mediators.

Methods: Using data from the Health and Retirement Study (HRS): Participant Lifestyle Questionnaire (2008-2010), odds ratio estimates of recovery were ascertained using binary logistic regression and post hoc Sobel tests were conducted to formally assess mediation.

Results: Net of demographic characteristics and socioeconomic status, increased neighborhood disorder was associated with lower odds of recovery. However, both psychosocial indicators and physical activity were significant individual partial mediators, which suggest neighborhood disorder influences recovery from physical impairment via psychosocial processes and barriers to physical activity.

Discussion: Reducing neighborhood disorder may enhance older residents’ psychosocial well-being and improve participation in physical activity, thus, increasing recovery from mobility limitation and preventing subsequent disability.
\end{abstract}

Keywords: neighborhood disorder; mobility limitation; recovery; disability; aging; Health and Retirement Study 
NEIGHBORHOOD DISORDER \& RECOVERY

\section{Introduction}

Mobility limitation, often conceptualized as lower-body impairment and operationalized as difficulty walking, climbing, or standing, is one of the most common forms of physical impairment among older adults. According to estimates from the National Health and Nutrition Examination Survey (NHANES), 30\% of Americans 60 years and older report difficulty walking a quarter mile, while 26\% report difficulty walking up 10 steps without resting (Ervin, 2006). Hardy et al. (2010) estimated, in 2003, over 15 million Medicare beneficiaries (65+ years) had difficulty walking a quarter of a mile. It is well established that mobility limitation predicts subsequent disability (i.e., difficulty completing essential tasks for daily living), hospitalization, and mortality (Hardy et al., 2011). There are also substantial healthcare costs associated with mobility limitation with additional total annual costs estimated to be over \$2,700 for older adults with walking difficulty (Hardy et al., 2011). Given the serious consequences of mobility limitation, it is important to explore potential facilitators of recovery including individual and environmental factors.

There is a growing body of literature linking the neighborhood context to maintaining mobility and functional health in later life (Beard et al., 2009; Glass \& Balfour, 2003). Compared with midlife adults, older adults spend more time in their neighborhood (Glass \& Balfour, 2003); therefore, the neighborhood context may hold important opportunities for intervention such as increasing rates of recovery. Accordingly, this research uses data from a nationally representative survey of older Americans to explore whether the neighborhood context, expressly physical neighborhood disorder, shapes recovery outcomes. Furthermore, we examine potential psychosocial (i.e., depressive symptomology and perceived mastery) and behavioral (i.e., participation in physical activity) mediators. As the US population continues to age, it is vital to for researchers, health professionals, and policymakers to consider upstream approaches to improving the functional health of older adults including the neighborhood or community level (Gehlert et al., 2008). This approach may address important health inequalities that stem from social and physical structures of neighborhoods, which could lead to a larger impact on older adults' health relative to focusing solely on individual factors related to mobility and functional health. 
NEIGHBORHOOD DISORDER \& RECOVERY

\section{Literature Review}

Neighborhoods and Functional Health among Older Adults

The neighborhood can be conceptualized as a "subsection of a larger community” (Sampson et al., 2002, 445). It is spatially defined and comprised of people and institutions influenced by ecological, cultural, and political factors (Sampson et al., 2002, p. 445). In relation to neighborhoods and health, Schulz and Northridge (2004, p. 457) identify three factors representing the various levels of health determinants including the fundamental (macro), intermediate (meso), and proximate (micro) determinants. The fundamental level includes the natural environment, macrosocial factors such as institutions, and inequalities, while the intermediate level includes the built environment and social environment (neighborhood/community context). The proximate level includes stressors, health behaviors, social integration and support. Neighborhood characteristics represent the intermediate or meso-level determinants of health and include both the social and the built/physical environment. The social environment reflects social capital (social ties/network/interactions), social norms and collective efficacy, community resources and investment, and routine activities (Sampson, Morenoff, \& GannonRowley, 2002), while the built environment includes "buildings, spaces, and products that are created or...modified by people” (Schulz \& Northridge, 2004, 456). The neighborhood context shapes exposure to stressors, social integration and support, and participation in health behaviors, which influences individual health outcomes. The various levels of the Schulz and Northridge model embody an upstream approach to health promotion, where addressing upstream (macro- and meso-level) factors are thought to create health-promoting opportunities down the stream at the proximate and individual-level (Berkman et al., 2000; Gehlert et al., 2008).

Schulz and Northridge’s (2004) model outlines the connections between the neighborhood context and an individual's health and well-being. The neighborhood holds the potential to impact health at every stage of the life course; however, Glass and Balfour (2003) suggest that neighborhoods may be particularly important for older individuals' functional health. Specifically, the authors note that older adults may experience increasing vulnerability from greater exposure to harmful environments and that 
the impact of this exposure may be exacerbated due to changes in biological susceptibility associated with aging. Additionally, the neighborhood context may be more salient for older individuals, versus younger individuals, because of a greater emphasis on aging in place and the corresponding "changing patterns of spatial use,” where older adults spend more time in their neighborhoods versus other environments such as work or school (Glass \& Balfour, 2003: 305). Indeed, previous empirical research underscores the significance of the neighborhood environment for functional health. Positive physical and social neighborhood characteristics such as the presence of sidewalks, good street lighting, cleanliness, and low fear of crime have been shown to be negatively associated with functional impairment, whereas socioeconomic disadvantage and negative neighborhood characteristics (e.g., increased physical disorder) are associated with more functional impairment (Balfour \& Kaplan, 2002; Beard et al., 2009; Clark et al., 2009; Clarke et al., 2011; Lang et al., 2008).

Neighborhood Disorder, Physical Health, \& Psychosocial Pathways

Assessments of physical neighborhood disorder (i.e., perceptions of vandalism/graffiti, rubbish/litter, vacant/deserted homes, and crime) may contribute to physical health via psychosocial and behavioral mechanisms. One suggested pathway from neighborhood disorder to physical health and mobility is through mental health and psychological distress (Hill, Ross, \& Angel, 2005). For example, numerous studies have found that persons living in neighborhoods with more disorder and incivilities are more likely to suffer from mental health disorders (Ross, 2000), such as depression and anxiety, and have lower levels of perceived mastery (Gilster, 2014; Schieman \& Meersman, 2004). Mair et al. (2010) observed that neighborhood stressors (e.g., perceived violence, physical decay, and disorder) were moderately associated with higher depression in both men and women. These disorders, in turn, have been found to significantly decrease physical activity and consequently mobility (de Wit et al., 2011). Burdette and Hill (2008) conceptualized psychological distress as a "lynchpin” mechanism linking neighborhood disorder to obesity by influencing physiological distress and found evidence to support this hypothesis; the authors suggested that high levels of disorder evoke feelings of threat and increases stress responses in the body — which harms physical health. 
Depressive symptomology as a measure of psychological distress may directly influence physical health via stress response; however, perceived mastery may act as a buffer against psychological distress and exert a protective effect on physical health by increasing “an individual's ability to cope with stressful demands” (Thoits, 2010, p. S46). An older adult’s ability to manage the psychological distress associated with neighborhood disorder may prevent (or facilitate recovery from) physical health declines by lowering the body's responses to stress and fear. Prior research illustrates the potential of neighborhood disorder to impact psychosocial measures of distress (depressive symptomology) as well as resources to cope with distress (perceived mastery), which shapes physical health outcomes directly via stress responses within the body.

Neighborhood Disorder, Physical Health, \& Barriers to Physical Activity

Neighborhood disorder may also influence physical health via behavioral mechanisms such as constraining physical activity within the neighborhood. Crime and fear of crime have been identified as potential link between neighborhood disorder and mental health and well-being (Lorenc et al., 2012); however, fear of crime may influence physical activity. While much of the literature focuses on direct effects of crime, such as physical injury and/or psychological trauma involved with being victimized, others have begun to focus on the indirect, neighborhood level effects through perceived environment and fear of crime (Lorenc et al., 2012). For example, both Gómez et al (2004) and McDonald (2008) found both direct and indirect exposure to violent crime to be associated with various negative health outcomes, notably poorer physical and psychological health. However, in looking at neighborhood level effects, Loukaitou-Sideris and Eck (2007) argue that fear inducing, built characteristics of neighborhood spaces, such as deteriorated buildings, graffiti, and empty lots, produce feelings of risk and fear, which can significantly alter one’s behaviors.

Lorenc et al. (2012) suggests that these feelings of risk and fear of crime may lead to "avoidance behaviors” such as limiting one’s movement outside the home, which may have an impact on both social interaction and physical activity in individuals. This fear forces individuals, particularly women and older adults, to restructure their lifestyles, predominantly related to outdoor physical activity, in order to avoid 
areas and activities they perceive as risky (Loukaitou-Sideris \& Eck, 2007). Similarly, Stafford et al. (2007) found that avoidance behaviors may limit interpersonal interaction, leading to poorer mental health and therefore reduced physical functioning. Relating to recovery explicitly, Latham and Clarke (2013) documented a positive association between higher self-ratings of perceived neighborhood safety and recovery from mobility limitation. Similar to Loukaitou-Sideris and Eck (2007) and Stafford and colleagues' (2007) contention, Latham and Clarke suggested that low levels of perceived neighborhood safety were a salient barrier to activity and engagement around the neighborhood, which reduced the odds of subsequent recovery from mobility limitation.

Further research suggests that neighborhood disorder may have an indirect effect on physical health by creating physical barriers to activity above and beyond avoidance behaviors, specifically, related to fear of crime. Neighborhoods with more disorder are often more economically disadvantage (Feldman \& Steptoe, 2004), which increases the likelihood these residents are living in areas with more physical barriers (Estabrooks, Lee, \& Gyurcsik, 2003). Rimmer (1999) argues that an important barrier to neighborhood-based physical activity for people with disabilities is access to built environments that support active options. For example, Spivock et al. (2008) found that the presence of "built environment buoys,” conceptualized as elements of the environment that support a person's involvement in physical activity despite the presence of functional limitation, was related to physical activity and active transportation among adults with mobility disabilities. King et al. (2011) found that older adults living in more walkable neighborhoods had more transport activity and moderate-to-vigorous physical activity than those living in less walkable neighborhoods. Specifically, the availability and condition of curb ramps and sidewalks, the presence and features of crosswalks, and the availability of resting places and shelters on streets, along with other physical features, have been noted as built environment activity barriers in studies of older adults with disabilities (Kirchner, Gerber, \& Smith, 2008, Rimmer et al., 2004, and Rosenberg et al., 2012). While physical neighborhood disorder is often thought to signify weak social control and increased threats related to social breakdown in communities (Burdette \& Hill, 2008; Ross \& Mirowsky, 1999), it may also be indicative of more physical barriers, which prevent subsequent 
activity—particularly among individuals with disabilities. Neighborhood disorder may prevent physical activity through both exposure to psychological threats (e.g., fear of crime) and physical barriers in the built environment, which, in turn, both lead to avoidance behaviors related to physical activity.

\section{Neighborhood Disorder and Recovery from Mobility Limitation}

The proximate mechanisms linking neighborhoods and physical health (above and beyond individual or compositional characteristics) are both psychosocial and behavioral. In general, research exploring the neighborhood and functional health tend to focus on the built environment and to a lesser extent the social environment. These studies often explore the relationship as mediated by psychosocial and/or physical barriers to physical activity (see Balfour \& Kaplan, 2002). Psychosocial pathways can directly influence functional health by impairing immune functioning as well as other physiological functions through stress responses, whereas behavioral pathways can directly influence functional health by preventing physical activity which is intimately tied to physical functioning. Previous research highlights that even minimal amounts of physical activity such as walking two blocks a day contribute positively to mobility and functional health among older adults (Simonsick et al., 2005). While other potential mediators linking neighborhood disorder to physical health may exist, psychosocial factors and physical activity are most represented in the current literature and appear to be dominant pathways; however, it is important to note that these two pathways do not exist independent from one another. Psychosocial factors and physical activity have the capacity to influence each other, yet the complex relationships among psychosocial factors and physical activity, as a whole, appear to link neighborhood disorder and functional health.

Nevertheless, the extant literature is limited in several ways because much of the research employs data from one US city such as Chicago, IL, Detroit, MI, Washington, DC, or Baltimore, MD, which limits generalizability, and neglects recovery as a potential functional health outcome. Compared with onset of functional impairment, recovery may be more sensitive to neighborhood context because additional stressors or perceived barriers may exacerbate the underlying condition(s) and constrain opportunities to regain physical functioning. To illustrate, Clarke et al. (2008) found that street and 
sidewalk conditions were predictive of outdoor mobility, but only among those respondents with severe impairment. The authors suggest that decreased lower-body strength and balance leads to more difficulty navigating physical obstacles outside, which influences whether older adults are active in their communities. Among older adults living with mobility limitation or disability, the neighborhood context—particularly physical neighborhood disorder — may have a larger impact on the decision to leave the home than among older adults free of impairment. Therefore, the neighborhood context may be a crucial upstream facilitator of recovery from mobility limitation.

Previous research delineates the significance of the neighborhood for older adults' functional health; however, recovery is typically overlooked in these studies, even though, the neighborhood environment appears to be more important for those with mobility limitation or disability. This research uniquely contributes to the current literature by examining whether physical neighborhood disorder predicts recovery from mobility limitation over a two-year period. Drawing on data from a nationally representative dataset, we investigate whether psychosocial factors (i.e., depressive symptomology and perceived mastery) and physical activity are salient mediators. Based upon prior research, we generated the following hypotheses:

Hypothesis 1: Net of demographic characteristics and socioeconomic status, greater physical neighborhood disorder will be associated with lower odds of recovery from mobility limitation.

Hypothesis 2: Depressive symptomology, perceived mastery, and physical activity will be significant individual partial mediators of physical neighborhood disorder and recovery from mobility limitation.

Hypothesis 3: As a group, the presumed mediators will explain a significant proportion of the total effect of neighborhood disorder on recovery from mobility limitation.

Figure 1 presents a conceptual model illustrating the potential mediating pathways between neighborhood disorder and recovery. We anticipate neighborhood disorder to be negatively associated with depressive symptomology, and positively associated with perceived mastery and physical activity. Additionally, we 
anticipate depressive symptomology to be negatively associated with recovery, whereas we expect perceived mastery and physical activity to be positively associated with recovery. By exploring salient mediators of physical neighborhood disorder and recovery, this research may inform upstream health promotion and interventions aimed at improving the functional health of older adults within their communities.

[Figure 1 About Here]

\section{Methods}

Data

Data for this research comes from the Health and Retirement Study (HRS): Psychosocial and Lifestyle Questionnaire (2008-2010), RAND HRS Data File (Version M), and the HRS 2012 (Early Version 1.0). The HRS is an ongoing nationally-representative longitudinal survey of Americans over the age of 50 (and their spouses) that uses a multi-stage, clustered probability frame. The Psychosocial and Lifestyle Questionnaire is a self-administered questionnaire that is left behind with the respondent following the face-to-face core interview. The goal of the Psychosocial and Lifestyle Questionnaire is to collect in-depth psychosocial data, and it is given to half-samples (i.e., 50\%) of the core respondents biennially (Smith et al., 2013). The first year of collection of psychosocial data was 2006 (with an online pilot in 2004). This research uses years 2008 (Wave 9) and 2010 (Wave 10) of the questionnaire because the prior collection episodes had important differences in measurement for key variables. For example, the independent variable of interest (i.e., neighborhood disorder) had reversed answer categories in 2006, which led to a lower Cronbach’s alpha score of 0.64 (Smith et al., 2013).

In 2008 (Wave 9), 6,884 respondents completed the Psychosocial and Lifestyle Questionnaire, and, in 2010 (Wave 10), 8,186 respondents completed the questionnaire. The response rate for the 2008 leave-behind questionnaire was approximately $89 \%$ among respondents who completed the core interview; the overall response rate was approximately 71\% (Smith et al., 2013). The response rates for 2010 were not available. The half-samples from 2008 and 2010 were pooled $(\mathrm{N}=15,070)$. The analytic sample was generated from these 15,000 respondents. Because this research is specifically interested in 
recovery from mobility limitation, only respondents reporting mobility impairment, which was nearly half of the original sample $(\mathrm{N}=7,379)$, during the year they completed the leave-behind questionnaire were included in the analytic sample. Analyses were run including and excluding respondents who died between follow-up waves. Both sets of models generated a similar pattern of results; therefore, for ease of interpretation only respondents who survived and provided valid data at both time points were included in the analytic sample (i.e., respondents with mobility limitation) $(\mathrm{N}=6,487)$. After omitting proxy interviews ( $<4 \%)$ and respondents with missing data $(<5 \%)$, the analytic sample was further reduced to a final sample size of 5,922. Proxy interviews were removed from the analyses because depressive symptomology was a key variable of interest and was not asked of proxy interviewees.

\section{Measures}

The dependent variable was recovery from mobility limitation over a two-year period. Mobility limitation was assessed using four lower-body functional limitation tasks: 1) walking several blocks; 2) walking one block; 3) climbing several flights of stairs; and 4) climbing one flight of stairs. Respondents reporting difficulty with any task were considered to have mobility limitation. Mobility limitation was measured the same year the respondents completed the leave-behind questionnaire (i.e., 2008 or 2010) using the RAND HRS functional limitation measures. A dichotomous recovery measure was created using the following wave of data. To illustrate, respondents who completed the leave-behind questionnaire in 2008 and reported having mobility limitation were then classified as having recovered if they reported no mobility limitation the following wave (i.e., 2010). Among respondents who completed the leave-behind questionnaire in 2010, the follow-up wave was collected in 2012. While a dichotomous variable was created for recovery where "0" is the reference category representing respondents who had persistent mobility limitation across the two waves and “ 1 ” was having mobility limitation, supplementary analyses employed a trichotomous measure of recovery where death and attrition were modeled using multinomial logistic regression. As mentioned earlier, the substantive findings from both modeling strategies were nearly identical; therefore, the results from the dichotomous measure of 
recovery are presented for ease of interpretation. (The results from multinomial logistic regression are available from the authors upon request.)

The independent variable of interest was physical neighborhood disorder. Physical neighborhood disorder was assessed using four indicators: 1) vandalism/graffiti; 2) rubbish/litter; 3) vacant/deserted homes; and 4) crime. Using a 7-point Likert scale, respondents were asked to rate their perceptions of their local area (i.e., everywhere within a 20 minute walk or about a mile). An index (range=1-7) was created by averaging the scores of the four neighborhood disorder items. Higher scores indicated more physical disorder, and respondents with two or more missing items (of the four items) were recoded as missing for the final index score. The physical neighborhood disorder items were adapted from the Project on Human Development in Chicago Neighborhoods (see Mendes de Leon et al., 2009) and the Cronbach’s Alpha was 0.83 in 2008 and 0.82 in 2010 (Smith et al., 2013).

Several demographic characteristics and socioeconomic status were included in the analyses as controls. Sex, age, race/ethnicity, and married/partner status were measured the same year the respondent completed the leave-behind questionnaire. A dichotomous measure of sex was created where female=1. Age was measured using a continuous measure of years and ranged from 51 to 101 . A four-category measure was created for race/ethnicity where White (reference), Black, Hispanic/Latino, and other race were the categories. A dichotomous measure of married/partner status was created where respondents reporting be married or living with a partner as if they were married (e.g., cohabitation)=1. Socioeconomic status was assessed using three measures: 1) educational attainment; 2) household income; and 3) household wealth. A trichotomous measure of educational attainment was created using self-reports of education credentials; the possible categories included having no high school degree, having a high school degree or equivalent (reference), and having a college degree (minimum of two-year degree/Associate degree). Both household income and household wealth were measured using quartiles. The bottom quartile was used as the reference category. Quartiles were based on the original half-samples (i.e., respondents with and without mobility limitation) in 2008 or 2010. 
One of the explicit goals of this research was to investigate potential mediators. Stemming from prior literature, we anticipated that psychosocial status (i.e., depressive symptomology and mastery) and physical activity would be salient mediators of neighborhood disorder and recovery from mobility limitation. Depressive symptomology was measured using an eight item version of the Center for Epidemiologic Studies Depression Scale (CESD). Respondents were asked whether in the past two weeks they felt: depressed, everything was an effort, lonely, sad, sleep was restless, could not get going, or enjoyed life. The two positive items (i.e., felt happy and enjoyed life) were reverse coded, and an index was created by summing the eight items. Higher values on the CESD reflected more depressive symptomology. Depressive symptomology was assessed during the HRS core interview, while mastery was measured using the leave-behind questionnaire. Perceived mastery was measured using a five item scale. Respondents were asked how much they agree or disagree (range=1 (strongly disagree) - 6 (strongly agree)) about the following statements: 1) I can do just about anything I really set my mind to; 2) When I really want to do something, I usually find a way to succeed; 3) Whether or not I am able to get what I want is in my own hands; 4) What happens to me in the future mostly depends on me; 5) I can do the things I want to do. The mastery scale was created by averaging the scores across all five items. Respondents missing three or more items were recoded as missing for the final value. The Cronbach's alpha was 0.89 in 2008 and 0.90 in 2010 (Smith et al., 2013).

Physical activity was assessed during the core interview. Respondents were asked the frequency in which they participate in light, moderate, and vigorous activity. The possible answer categories included never (=0), 1-3 times per month (=1), 1 time per week $(=2)$, more than 2 times per week $(=3)$, and every day (=4). A physical activity index was created by weighting the type of physical activity by intensity (i.e., low intensity=1.2; moderate intensity=1.4; vigorous intensity=1.8) and then summing the scores for all three intensity levels. The physical activity index ranged from 0 (no physical activity) to 17.6 (low, moderate, and vigorous activity every day). The intensity weights were motivated by metabolic equivalent (MET) recommendations (i.e., light=2; moderate=4; vigorous=8) (see Meyer, Janke, \& Beaujean, 2014); however, when indexed using the original MET recommendations, there was a wide 
range (0-56), which created small estimates and increased skewness. Therefore, we elected to scale the weights, which improved overall model fit in supplementary analyses (not shown).

\section{Analytic Strategy}

Because we are interested in exploring potential mediators of neighborhood disorder and recovery, we must first establish bivariate correlations among the independent variable of interest, presumed mediators, and dependent variable. Table 2 presents the bivariate associations for the focal variables. Next, a series of logistic regression models (see Table 3) were created to ascertain the odds ratio estimates and unstandardized coefficients of recovery over a two-year period. In total six models are presented with Model 1 (adjusted for demographic characteristics) and Model 2 (adjusted for socioeconomic status) illustrating the association between physical neighborhood disorder and recovery from mobility limitation. Models 3-5 individually introduce the three presumed mediators (i.e., depressive symptomology, mastery, and physical activity), while Model 6 includes all three potential mediators as a multiple mediator model. Additionally, since the HRS includes data from multiple respondents within a household (i.e., spouses), robust standard errors were employed to account for potential clustering at the household level.

To formally test whether depressive symptomology, mastery, and physical activity were significant individual partial mediators, post hoc Sobel tests of mediation for binary logistic regression were conducted. Sobel test of mediation is a conservative test of mediation (MacKinnon, Warsi, \& Dwyer, 1995) and requires information from two sets of models—specifically testing the $X \rightarrow Y$ and $\mathrm{X} \rightarrow \mathrm{M}$ relationships, where $\mathrm{M}$ is the presumed mediator. Because the outcome variable is a binary measure and the independent variable of interest and presumed mediators are treated as continuous measures, the Sobel tests must be adjusted so that coefficients are comparable across the models (for an overview see MacKinnon \& Dwyer, 1993). To handle the different scales between binary logistic regression and ordinary least squares (OLS) linear regression, the unstandardized coefficients from both logistic and linear regression were multiplied by their standard deviation and divided by the standard 
NEIGHBORHOOD DISORDER \& RECOVERY

deviation of the outcome variable (recovery). Comparable standard errors were generated using the same method.

\section{Results}

\section{Sample Characteristics}

Table 1 presents the descriptive statistics for the analytic sample ( $\mathrm{N}=5,922)$. Approximately $17 \%$ of the sample recovered from mobility limitation over the two-year interval. The average physical neighborhood disorder score was 2.7, suggesting most respondents experienced low neighborhood disorder. Two-thirds of the analytic sample was female, and the average age was about 70 years. The majority of the analytic sample was White (72.9\%) and married or partnered (59.6\%). Most respondents had a high school degree (58.3\%), and the modal category for household income and wealth was the bottom quartile. Because the analytic sample represents respondents with mobility limitation, it is to be expected that there are more low socioeconomic status respondents compared with the original sample (i.e., respondents with and without mobility limitation). On average, respondents in the analytic sample reported about two depressive symptoms and a perceived mastery score of 4.6. The average physical activity score was 6.2, which corresponds to, roughly, weekly physical activity of lower intensity. Finally, approximately $46 \%$ of the respondents completed the leave-behind questionnaire in 2008meaning a little more than half of the respondents completed the leave-behind questionnaire in 2010.

[Insert Table 1 About Here]

\section{Bivariate Correlations}

Baron and Kenny (1986) outline four criteria necessary for investigating mediation including: 1) a significant correlation between the independent and dependent (outcome) variable; 2) a significant correlation between the independent variable and presumed mediator(s); 3) the mediator affects the dependent variable, net of the independent variable; and 4) a reduction in the strength in the association between the independent variable of interest and dependent variable, net of the presumed mediator(s). To address the first two criteria, we examined the bivariate correlations among our focal variables. In Table 2, the Pearson's $r$ values are presented for the outcome variable (i.e., recovery from mobility limitation), 
the independent variable of interest (i.e., perceived neighborhood disorder), and the potential mediators (i.e., depressive symptomology, perceived mastery, and physical activity). All correlations were significant at an alpha-level of less than 0.01. There was a significant (albeit weak) negative correlation between the independent variable (neighborhood disorder) and dependent variable (recovery).

Additionally, all three potential mediators were significantly correlated with the neighborhood disorder; depressively symptomology was positively associated with neighborhood disorder, while perceived mastery and physical activity were both negatively associated with neighborhood disorder.

\section{[Insert Table 2 About Here]}

To establish the third and fourth Baron and Kenny (1986) mediation criteria, a series of models from binary logistic regression were generated to assess whether physical neighborhood disorder was a significant predictor of recovery and whether that relationship was reduced with the introduction of the presumed mediators. Post hoc Sobel tests of mediation were then conducted to formally test for partial mediation of the presumed mediators. This included generating three sets of models (not shown, but available from the authors upon request) from OLS linear regression that predicted $X \rightarrow M$ (i.e., physical neighborhood disorder on each of the presumed mediators). For ease of interpretation, Table 3 presents the Odds Ratio (OR) estimates and unstandardized coefficients of recovery from mobility limitation; however, the formal tests of mediation were conducted using standardized coefficients.

In Model 1, physical neighborhood disorder (OR=0.91) was significantly associated with recovery, net of demographic characteristics. For every additional point on the physical neighborhood disorder scale, the odds of recovery was reduced by approximately $9 \%$. To further illustrate the size of the effect of physical neighborhood disorder, predicted probabilities were calculated. Among respondents reporting the lowest physical neighborhood disorder (=1), the probability of recovery was $19.6 \%$, while, among respondents reporting the highest amount of physical disorder (=7), the probability of recovery was $11.9 \%$. The difference in predicted probabilities from the lowest to highest neighborhood disorder represented a near $40 \%$ change in probability of recovery. Among demographic characteristics, sex $(\mathrm{OR}=0.84)$, age $(\mathrm{OR}=0.98)$, and marital/partner status $(\mathrm{OR}=1.40)$ were significant predictors of recovery. 
Women and older respondents had lower odds of recovery, while married/partnered respondents had greater odds of recovery.

\section{[Insert Table 3 About Here]}

Model 2 further adjusts for socioeconomic status. Physical neighborhood disorder (OR=0.94) remained significant with the introduction of socioeconomic status, although, the strength of the associated was attenuated. Net of demographic characteristics and socioeconomic status, on average, respondents experienced a $6 \%$ reduction in the odds of recovery from mobility limitation for every additional point on the physical neighborhood disorder scale. Sex and age remained relatively stable from Model 1 to Model 2. Marital/partner status was no longer a significant predictor of recovery, whereas race/ethnicity became a significant predictor of recovery after adjusting for socioeconomic status. Relative to White respondents, Black (OR=1.43), Hispanic/Latino (OR=1.33), and other race (OR=1.64) respondents had greater odds of recovery. Having a college degree $(\mathrm{OR}=1.20)$ was associated with increased odds of recovery. Higher incomes, compared with the bottom income quartile, were also associated with increased odds of recovery. Respondents in the top two wealth quartiles had improved odds of recovery, relative to the bottom wealth quartile.

With the introduction of depressive symptomology (Model 3), physical neighborhood disorder was no longer a significant predictor of recovery from mobility limitation, which suggests that depressive symptomology partially mediated the association. As anticipated, depressive symptomology (OR=0.84) was a significant predictor of recovery. For every additional depressive symptom reported, the odds of recovery was decreased by $16 \%$. A Sobel test of mediation for binary logistic regression provides strong evidence that depressive symptomology was a partial mediator with a $z$-score of $-4.72(p<0.001)$. After the standardizing the coefficients, the indirect effect was -0.015 and total effect was -0.059 ; therefore, approximately $26 \%$ of the effect of physical neighborhood disorder on recovery from mobility limitation was mediated by depressive symptomology.

Model 4 included physical neighborhood disorder, demographic characteristics, socioeconomic status, and introduced perceived mastery. Similarly to depressive symptomology, with the inclusion of 
perceived mastery, physical neighborhood disorder was no longer a significant predictor at an alpha-level of less than 0.05 . Perceived mastery $(\mathrm{OR}=1.29)$ was positively associated with recovery. For every additional point on the perceived mastery scale, there was a $29 \%$ improvement in the odds of recovery from mobility limitation. Again, the Sobel test of mediation provided strong evidence of partial mediation for perceived mastery with a $z$-score of $-5.62(p<0.001)$. Approximately $32 \%$ of the effect of physical neighborhood disorder on recovery from mobility limitation (total effect $=-0.044$ ) was mediated by perceived mastery (indirect effect=-0.014).

Physical activity was incorporated in Model 5 (without the other two psychosocial mediators). In Model 5, physical neighborhood disorder was no longer a significant predictor. The Sobel test of mediation generated a $z$-score of $-3.45(p<0.001)$, which suggests that physical activity was also significant partial mediator of physical neighborhood disorder and recovery. Approximately $38 \%$ of the effect of physical neighborhood disorder on recovery from mobility limitation (total effect=-0.060) was mediated (indirect effect=-0.023) by perceived mastery.

Each of the individual mediators were significant partial mediators; however, the three presumed mediators were also associated with each other as evidenced by bivariate correlations (see Table 2). Therefore, the final model (Model 6) included all three presumed mediators so that the independent effect of each mediator on recovery could be assessed. Physical neighborhood disorder continued to be insignificant and was further attenuated with the inclusion of all three presumed mediators. Additionally, depressive symptomology $(\mathrm{OR}=0.88)$, perceived mastery $(\mathrm{OR}=1.18)$ and physical activity $(\mathrm{OR}=1.10)$ remained significant predictors of recovery.

\section{[Table 4 About Here]}

Using the product of coefficients method for multiple mediators (for an overview see Preacher \& Hayes, 2008), we formally tested whether the total indirect effect of all three presumed mediators was significant. Table 4 presents the unstandardized coefficients and standard errors, generated from both binary logistic regression and OLS regression, that were used to estimate the total indirect effect in the multiple mediator model. With a $z$-score of -5.66 ( $p$-value $<0.001$ ), we were able to reject the null 
hypothesis that the total indirect effect was zero, which suggests that the presumed mediators explained a significant proportion of the total effect of neighborhood disorder on recovery. Furthermore, the product of coefficients method allowed for a closer examination of each presumed mediator. In the multiple mediator model, only physical activity $(z$-score=-2.38, $p$-value=0.02) was a significant mediator at an alpha-level of less than 0.05; however, perceived mastery ( $z$-score=-1.69, $p$-value=0.09) was a significant mediator at an alpha-level of less than 0.10 . There was strong evidence that physical activity was a salient mediator of neighborhood disorder and recovery and some evidence that perceived mastery was also an important mediator in the multiple mediator model.

\section{Discussion}

Drawing from extant literature, we hypothesized that physical neighborhood disorder would be negatively associated with recovery from mobility limitation among older adults, net of demographic characteristics and socioeconomic status. The foregoing analyses provide evidence in support of this hypothesis. Higher levels of physical neighborhood disorder reduced the odds of recovery over a two-year period. Additionally, we hypothesized that association between physical neighborhood disorder and recovery from mobility limitation was mediated by psychosocial processes and barriers to physical activity. We tested this hypothesis by examining whether depressive symptomology, perceived mastery, and physical activity were significant $(\alpha<0.05)$ individual partial mediators. Likewise, this hypothesis was supported by our results. Additionally, we hypothesized that all three presumed mediators, as a group, would explained a significant proportion of the total effect. Again, there was evidence to support that psychosocial factors and physical activity, together, explained a significant portion of the association between neighborhood disorder and recovery. When the multiple mediators were assessed simultaneously, there was evidence that physical activity and perceived mastery were salient mediators. These findings suggest that reducing physical neighborhood disorder may lead to increased physical activity as well as improved mental health such as increased perceived mastery among older residents, which could improve recovery outcomes and functional health within communities. 
NEIGHBORHOOD DISORDER \& RECOVERY

\section{Limitations}

A potential limitation of this research is the use of subjective assessments of physical neighborhood disorder; however, we argue that subjective assessments are well-suited for these analyses. While objective measures are typically preferred by researchers, subjective neighborhood assessments are valuable because perception may be more powerful for functional health and mobility, especially among older adults. Parra et al. (2010) and Hoehner et al. (2005) found that perceptions of environment were more predictive of health outcomes than objective measures of environment. There is evidence that perceived neighborhood deterioration is a stronger predictor of fear of crime than objective measures of neighborhood deterioration (Perkins \& Taylor, 1996). Clark et al. (2009) found that, in examining both objective and subjective safety/crime measures, only perceptions of neighborhood safety were correlated with mobility disability. Although we maintain that subjective assessments are advantageous for exploring the link between neighborhoods and older adults' functional health, we recognize that the policy implications are more ambiguous because the objective neighborhood factors shaping older residents’ perceptions are not directly measured. Furthermore, because psychosocial factors may bias subjective assessments of neighborhood disorder, use of these measures may have introduced samesource bias in these results.

Additionally, we cannot discount reverse causation in relation to physical neighborhood disorder and the key mediators, which were measured at the same time. It is conceivable that certain psychosocial and physical activity histories lead to circumstances where older adults are exposed to (or perceive) more disordered neighborhoods, instead of neighborhood disorder shaping depressive symptomology, perceived mastery, and physical activity. However, the alternative would most likely operate through socioeconomic status. To illustrate, low socioeconomic neighborhoods are more likely show signs of physical neighborhood disorder (Latkin \& Curry, 2003), and individuals with a history of mental health conditions (such as depression) or low mastery are more likely to have lower socioeconomic status (McLeod \& Kessler, 1990; Miech \& Shanahan, 2000). To address this issue, we employ multiple socioeconomic measures including education, household income, and household wealth, yet we cannot 
state with certainty that neighborhood disorder precedes the exposure to the proximate mediators, which limits us from making causal inferences with this data.

Finally, another limitation of this research is our inability to disentangle the temporal ordering of all three presumed mediators. Psychosocial factors and physical activity are intimately linked. All three mediators were correlated; therefore, we are unable to draw conclusions about which mediator precedes one another. Our goal was to examine the most commonly cited mediators, which conceptually represented dominant pathways linking neighborhood disorder to recovery from mobility limitation; however, future research, using longitudinal data, may want to further explore these potential pathways including examining the importance of timing and cumulative burden of each mediator. It is possible that these mediators are also moderators. It was not within the scope of this research to explore moderation, but it may be another fruitful avenue of research with important policy implications.

While this research has several caveats, the findings still provide support that the neighborhood context influences recovery from mobility limitation through psychosocial factors and by exposing residents to facilitators or barriers to physical activity. In relation to policy implications, these findings suggest that improvements to the physical neighborhood such as reducing graffiti and the number of vacant homes may encourage recovery among older adults living with mobility limitation. This type of intervention represents an upstream approach, which may impact downstream mechanisms such as mental health, health behaviors, and well-being. Branas et al. (2011) provide an applied example of this approach; the authors conducted a decade long difference-in-difference analysis of greening vacant urban space and observed lower crime rates among neighborhoods exposed to the greening program.

Furthermore, the residents in the treatment neighborhoods reported lower levels of stress and increased exercise (Branas et al., 2011).

As the US population continues to age, policymakers interested in decreasing disability and the healthcare costs associated with disability will need to adopt upstream approaches to functional health promotion and intervention to see meaningful changes within communities. Furthermore, the burden of disability among older adults is not distributed equally; older adults with low socioeconomic status and 
racial minorities experience higher rates of disability in later life (Thorpe et al., 2011). These older individuals are also more likely to be exposed to harmful neighborhood environments (Latkin \& Curry, 2003). Thus, adopting upstream approaches that address intermediate or meso-level determinants of health such as the neighbor context may reduce functional health disparities among older adults. This research purposely examined recovery from mobility limitation as an outcome and documented the importance of the neighborhood context. Mobility limitation is one of the most common types of impairment among older adults and improving the neighborhood environment may especially impact older adults living with mobility impairment. However, like many age-friendly policies, reducing physical neighborhood disorder would be beneficial for the physical and mental health of all residents, young and old, with and without impairment. 
NEIGHBORHOOD DISORDER \& RECOVERY

\section{References}

Balfour, J. L., \& Kaplan, G. A. (2002). Neighborhood Environment and Loss of Physical Function in Older Adults: Evidence from the Alameda County Study. American Journal of Epidemiology, 155(6), 507-515. doi: 10.1093/aje/155.6.507

Baron, R. M., \& Kenny, D. A. (1986). The moderator-mediator variable distinction in social psychological research: Conceptual, strategic and statistical considerations. Journal of Personality and Social Psychology, 51, 1173-1182.

Beard, J. R., Blaney, S., Cerda, M., Frye, V., Lovasi, G. S., Ompad, D., ... \& Vlahov, D. (2009). Neighborhood characteristics and disability in older adults. The Journals of Gerontology Series B: Psychological Sciences and Social Sciences, gbn018.

Berkman, L. F., Glass, T., Brissette, I., \& Seeman, T. E. (2000). From social integration to health: Durkheim in the new millennium. Social science \& medicine, 51(6), 843-857.

Branas, C. C., Cheney, R. A., MacDonald, J. M., Tam, V. W., Jackson, T. D., \& Ten Have, T. R. (2011). A difference-in-differences analysis of health, safety, and greening vacant urban space. American Journal of Epidemiology, kwr273.

Burdette, A. M., \& Hill, T. D. (2008). An examination of processes linking perceived neighborhood disorder and obesity. Social Science \& Medicine, 67(1), 38-46.

Clark, C., Kawachi, I., Ryan, L., Ertel, K., Fay, M., \& Berkman, L. (2009). Perceived neighborhood safety and incident mobility disability among elders: the hazards of poverty. BMC Public Health, 9(1), 162.

Clarke, P., \& Gallagher, N. (2013). Optimizing Mobility in Later Life: The Role of the Urban Built Environment for Older Adults Aging in Place. Journal of Urban Health, 1-13. doi: 10.1007/s11524-013-9800-4

de Wit, L. M., Fokkema, M., van Straten, A., Lamers, F., Cuijpers, P., \& Penninx, B. W. (2010). Depressive and anxiety disorders and the association with obesity, physical, and social activities. Depression and anxiety, 27(11), 1057-1065. 
Ervin R.B. (2006) Prevalence of functional limitations among adults 60 years of age and over: United States, 1999-2002. Advance data from vital and health statistics; no 375. Hyattsville,MD: National Center for Health Statistics.

Estabrooks, P. A., Lee, R. E., \& Gyurcsik, N. C. (2003). Resources for physical activity participation: does availability and accessibility differ by neighborhood socioeconomic status?. Annals of Behavioral Medicine, 25(2), 100-104.

Feldman, P. J., \& Steptoe, A. (2004). How neighborhoods and physical functioning are related: the roles of neighborhood socioeconomic status, perceived neighborhood strain, and individual health risk factors. Annals of Behavioral Medicine, 27(2), 91-99.

Gilster, M. E. (2014). Neighborhood Stressors, Mastery, and Depressive Symptoms: Racial and Ethnic Differences in an Ecological Model of the Stress Process in Chicago. Journal of Urban Health, 117.

Gehlert, S., Sohmer, D., Sacks, T., Mininger, C., McClintock, M., \& Olopade, O. (2008). Targeting health disparities: A model linking upstream determinants to downstream interventions. Health Affairs, 27(2), 339-349.

Glass, T. A., \& Balfour, J. L. (2003). Neighborhoods, aging, and functional limitations. Neighborhoods and health, 303-334.

Hardy, S. E., Kang, Y., Studenski, S. A., \& Degenholtz, H. B. (2011). Ability to walk 1/4 mile predicts subsequent disability, mortality, and health care costs. Journal of general internal medicine, 26(2), 130-135.

Hardy, S. E., McGurl, D. J., Studenski, S. A., \& Degenholtz, H. B. (2010). Biopsychosocial Characteristics of Community-Dwelling Older Adults with Limited Ability to Walk One-Quarter of a Mile. Journal of the American Geriatrics Society, 58(3), 539-544.

Health and Retirement Study (HRS), public use dataset. (2012). Produced and distributed by the University of Michigan with funding from the National Institute on Aging (grant number NIA U01AG009740). Ann Arbor, MI. 
Hill, T. D., Ross, C. E., \& Angel, R. J. (2005). Neighborhood disorder, psychophysiological distress, and health. Journal of Health and Social Behavior, 46(2), 170-186.

Hoehner, C. M., Brennan Ramirez, L. K., Elliott, M. B., Handy, S. L., \& Brownson, R. C. (2005).

Perceived and objective environmental measures and physical activity among urban adults.

American Journal of Preventive Medicine, 28(2, Supplement 2), 105-116. doi:

http://dx.doi.org/10.1016/j.amepre.2004.10.023

King, A. C., Sallis, J. F., Frank, L. D., Saelens, B. E., Cain, K., Conway, T. L., Chapman, J.E., Ahn, D.K., \& Kerr, J. (2011). Aging in neighborhoods differing in walkability and income: associations with physical activity and obesity in older adults. Social Science \& Medicine, 73(10), 1525-1533.

Kirchner, C. E., Gerber, E. G., \& Smith, B. C. (2008). Designed to deter: community barriers to physical activity for people with visual or motor impairments. American Journal of Preventive Medicine, 34(4), 349-352.

Lang, I. A., Llewellyn, D. J., Langa, K. M., Wallace, R. B., \& Melzer, D. (2008). Neighbourhood deprivation and incident mobility disability in older adults. Age and Ageing, 37(4), 403-410. doi: 10.1093/ageing/afn092

Latkin, C. A., \& Curry, A. D. (2003). Stressful neighborhoods and depression: a prospective study of the impact of neighborhood disorder. Journal of health and social behavior, 34-44.

Latham, K., \& Clarke, P. J. (2013). The Role of Neighborhood Safety in Recovery From Mobility Limitations Findings From a National Sample of Older Americans (1996-2008). Research on aging, 35(4), 481-502.

Lorenc, T., Clayton, S., Neary, D., Whitehead, M., Petticrew, M., Thomson, H., Cummins S., Sowden A. \& Renton, A. (2012). Crime, fear of crime, environment, and mental health and wellbeing: Mapping review of theories and causal pathways. Health \& place, 18(4), 757-765.

Loukaitou-Sideris, A., \& Eck, J. E. (2007). Crime prevention and active living. American Journal of Health Promotion, 21(4s), 380-389. 
MacKinnon, D. P., \& Dwyer, J. H. (1993). Estimating mediated effects in prevention studies. Evaluation review, 17(2), 144-158.

MacKinnon, D. P., Warsi, G., \& Dwyer, J. H. (1995). A simulation study of mediated effect measures. Multivariate Behavioral Research, 30, 41-62.

Mair, C., Diez Roux, A. V., \& Morenoff, J. D. (2010). Neighborhood stressors and social support as predictors of depressive symptoms in the Chicago Community Adult Health Study. Health \& place, 16(5), 811-819.

McDonald, N. C. (2008). The effect of objectively measured crime on walking in minority adults. American Journal of Health Promotion, 22(6), 433-436.

McLeod, J. D., \& Kessler, R. C. (1990). Socioeconomic status differences in vulnerability to undesirable life events. Journal of health and social behavior, 162-172.

Mendes de Leon, C. F., Cagney, K. A., Bienias, J. L., Barnes, L. L., Skarupski, K. A., Scherr, P. A., \& Evans, D. A. (2009). Neighborhood Social Cohesion and Disorder in Relation to Walking in Community-Dwelling Older Adults A Multilevel Analysis. Journal of aging and health, 21(1), 155-171.

Miech, R. A., \& Shanahan, M. J. (2000). Socioeconomic status and depression over the life course. Journal of Health and Social Behavior, 162-176.

Meyer, M. R. U., Janke, M. C., \& Beaujean, A. A. (2014). Predictors of older adults’ personal and community mobility: Using a comprehensive theoretical mobility framework. The Gerontologist, 54(3), 398-408.

Parra, D. C., Gomez, L. F., Sarmiento, O. L., Buchner, D., Brownson, R., Schimd, T., .. . Lobelo, F. (2010). Perceived and objective neighborhood environment attributes and health related quality of life among the elderly in Bogota, Colombia. Social Science \& Medicine, 70(7), 1070-1076.

Preacher, K. J., \& Hayes, A. F. (2008). Asymptotic and resampling strategies for assessing and comparing indirect effects in multiple mediator models. Behavior research methods, 40(3), 879-891.

RAND HRS Data, Version M. (2012). Produced by the RAND Center for the Study of Aging, with funding from the National Institute on Aging and the Social Security Administration. Santa 
Monica, CA.

Rimmer, J. H. (1999). Health promotion for people with disabilities: the emerging paradigm shift from disability prevention to prevention of secondary conditions. Physical therapy, 79(5), 495-502.

Ross, C. E. (2000). Neighborhood disadvantage and adult depression. Journal of Health and Social Behavior, 177-187.

Ross, C. E., \& Mirowsky, J. (1999). Disorder and decay the concept and measurement of perceived neighborhood disorder. Urban Affairs Review, 34(3), 412-432.

Rosenberg, D. E., Huang, D. L., Simonovich, S. D., \& Belza, B. (2012). Outdoor built environment barriers and facilitators to activity among midlife and older adults with mobility disabilities. The Gerontologist, gns119.

Sampson, R. J., Morenoff, J. D., \& Gannon-Rowley, T. (2002). Assessing" neighborhood effects": Social processes and new directions in research. Annual review of sociology, 443-478.

Schieman, S., \& Meersman, S. C. (2004). Neighborhood problems and health among older adults: received and donated social support and the sense of mastery as effect modifiers. The Journals of Gerontology Series B: Psychological Sciences and Social Sciences, 59(2), S89-S97.

Schulz, A., \& Northridge, M. E. (2004). Social determinants of health: implications for environmental health promotion. Health Education \& Behavior, 31(4), 455-471.

Simonsick, E. M., Guralnik, J. M., Volpato, S., Balfour, J., \& Fried, L. P. (2005). Just get out the door! Importance of walking outside the home for maintaining mobility: findings from the women's health and aging study. Journal of the American Geriatrics Society, 53(2), 198-203.

Smith, J., G. Fisher, L. Ryan, P. Clarke, J. House, \& D. Weir. 2013. Psychosocial and lifestyle questionnaire 2006-2010. Survey Research Center, Institute of Social Research, University of Michigan: Ann Arbor, MI. http://hrsonline.isr.umich.edu/sitedocs/userg/HRS2006-

\section{SAQdoc.pdf}


Spivock, M., Gauvin, L., Riva, M., \& Brodeur, J. M. (2008). Promoting active living among people with physical disabilities: evidence for neighborhood-level buoys. American Journal of Preventive Medicine, 34(4), 291-298.

Stafford, M., Chandola, T., \& Marmot, M. (2007). Association between fear of crime and mental health and physical functioning. Journal Information, 97(11).

Thorpe, R. J., Koster, A., Kritchevsky, S. B., Newman, A. B., Harris, T., Ayonayon, H. N., ... \& Simonsick, E. M. (2011). Race, socioeconomic resources, and late-life mobility and decline: Findings from the Health, Aging, and Body Composition Study. The Journals of Gerontology Series A: Biological Sciences and Medical Sciences, glr102.

Thoits, P. A. (2010). Stress and health major findings and policy implications. Journal of health and social behavior, 51(1 suppl), S41-S53. 
Table 1. Descriptive Statistics of the Analytic Sample (i.e., Respondents with Mobility Limitation) $(\mathrm{N}=5,922)$

\begin{tabular}{lc}
\hline & $\begin{array}{c}\text { Distributional } \\
\text { Information }\end{array}$ \\
\hline Recovered from Mobility Limitation & $17.1 \%$ \\
Physical Neighborhood Disorder & $2.7(1.5)$ \\
Demographic Characteristics: & \\
Sex (female=1) & $66.6 \%$ \\
Age (years) & $69.8(9.9)$ \\
Race/Ethnicity: & \\
White & $72.9 \%$ \\
Black & $15.7 \%$ \\
Hispanic/Latino & $9.8 \%$ \\
Other Race & $1.6 \%$ \\
Married/Partnered (=1) & $59.6 \%$ \\
Socioeconomic Status: & \\
Education: & \\
No High School Degree & $21.0 \%$ \\
High School Degree & $58.3 \%$ \\
College Degree & $20.7 \%$ \\
Income Quartiles: & \\
Bottom Quartile & $30.6 \%$ \\
Lower Middle Quartile & $28.2 \%$ \\
Upper Middle Quartile & $24.6 \%$ \\
Upper Quartile & $16.6 \%$ \\
Wealth Quartiles: & \\
Bottom Quartile & $31.7 \%$ \\
Lower Middle Quartile & $27.0 \%$ \\
Upper Middle Quartile & $22.8 \%$ \\
Upper Quartile & $18.5 \%$ \\
Perceived Mastery & $4.6(1.1)$ \\
Depressive Symptomology & $1.9(2.2)$ \\
Physical Activity & $6.2(4.0)$ \\
2008 Survey Year (=1) & $45.6 \%$ \\
\hline
\end{tabular}

Notes: aPercentage distributions are shown for categorical variables; means and (standard deviations) are shown for continuous variables. 
Table 2. Bivariate correlations among focal variables $(\mathrm{N}=5,922)$

\begin{tabular}{lccccc}
\hline & $\begin{array}{c}\text { A. Recovery } \\
\text { from Mobility } \\
\text { Limitation }\end{array}$ & $\begin{array}{c}\text { B. Perceived } \\
\text { Neighborhood } \\
\text { Disorder }\end{array}$ & $\begin{array}{c}\text { C. Depressive } \\
\text { Symptoms }\end{array}$ & $\begin{array}{c}\text { D. Perceived } \\
\text { Mastery }\end{array}$ & $\begin{array}{c}\text { E. Physical } \\
\text { Activity }\end{array}$ \\
\hline A. & -- & $-0.04^{* *}$ & $-0.13^{* * *}$ & $0.12^{* * *}$ & $0.18^{* * *}$ \\
B. & -- & -- & $0.14^{* * *}$ & $-0.13^{* * *}$ & $-0.08^{* * *}$ \\
C. & -- & -- & -- & $-0.24^{* * *}$ & $-0.19^{* * *}$ \\
D. & -- & -- & -- & -- & $0.17^{* * *}$ \\
E. & -- & -- & -- & -- \\
\hline
\end{tabular}

Notes: ${ }^{*} \mathrm{p} \leq 0.05 ;{ }^{* *} \mathrm{p} \leq 0.01 ; * * * \mathrm{p} \leq 0.001$ 
Table 3. Odds Ratio Estimates and Unstandardized Binary Logistic Regression Coefficients (and Standard Errors) of Recovery from Mobility Limitation $(\mathrm{N}=5,922)$

\begin{tabular}{|c|c|c|c|c|c|c|}
\hline & Model 1 & Model 2 & Model 3 & Model 4 & Model 5 & Model 6 \\
\hline Physical Neighborhood Disorder & $\begin{array}{l}0.91 * * * \\
-0.10(0.03)\end{array}$ & $\begin{array}{l}0.94^{*} \\
-0.06(0.03)\end{array}$ & $\begin{array}{l}0.96 \\
-0.04(0.03)\end{array}$ & $\begin{array}{l}0.96 \\
-0.04(0.03)\end{array}$ & $\begin{array}{l}0.96 \\
-0.05(0.03)\end{array}$ & $\begin{array}{l}0.98 \\
-0.02(0.03)\end{array}$ \\
\hline \multicolumn{7}{|l|}{ Demographic Characteristics: } \\
\hline Sex $($ female $=1)$ & $\begin{array}{l}0.84^{*} \\
-0.18(0.07)\end{array}$ & $\begin{array}{l}0.84^{*} \\
-0.18(0.08)\end{array}$ & $\begin{array}{l}0.85^{*} \\
-0.17(0.08)\end{array}$ & $\begin{array}{l}0.84^{*} \\
-0.18(0.08)\end{array}$ & $\begin{array}{l}0.81 * * \\
-0.21(0.08)\end{array}$ & $\begin{array}{l}0.83^{*} \\
-0.19(0.08)\end{array}$ \\
\hline Age (years) & $\begin{array}{l}0.98 * * * \\
-0.02(0.00)\end{array}$ & $\begin{array}{l}0.97 * * * \\
-0.03(0.00)\end{array}$ & $\begin{array}{l}0.97 * * * \\
-0.03(0.00)\end{array}$ & $\begin{array}{l}0.97 * * * \\
-0.03(0.00)\end{array}$ & $\begin{array}{l}0.98 * * * \\
-0.02(0.00)\end{array}$ & $\begin{array}{l}0.97 * * * \\
-0.03(0.00)\end{array}$ \\
\hline \multicolumn{7}{|l|}{$\begin{array}{l}\text { Race/Ethnicity: } \\
\text { White (ref.) }\end{array}$} \\
\hline Black & $\begin{array}{l}1.15 \\
0.14(0.10)\end{array}$ & $\begin{array}{l}1.43 * * * \\
0.36(0.11)\end{array}$ & $\begin{array}{l}1.41 * * \\
0.34(0.11)\end{array}$ & $\begin{array}{l}1.37 * * \\
0.31(0.11)\end{array}$ & $\begin{array}{l}1.51^{* *} \\
0.41(0.11)\end{array}$ & $\begin{array}{l}1.44 * * * \\
0.37(0.11)\end{array}$ \\
\hline Hispanic/Latino & $\begin{array}{l}0.97 \\
-0.03(0.12)\end{array}$ & $\begin{array}{l}1.33^{*} \\
0.29(0.13)\end{array}$ & $\begin{array}{l}1.44 * * \\
0.37(0.13)\end{array}$ & $\begin{array}{l}1.30 * \\
0.26(0.13)\end{array}$ & $\begin{array}{l}1.27 \\
0.24(0.13)\end{array}$ & $\begin{array}{l}1.34 * \\
0.29(0.13)\end{array}$ \\
\hline Other Race & $\begin{array}{l}1.40 \\
0.33(0.24)\end{array}$ & $\begin{array}{l}1.64^{*} \\
0.50(0.25)\end{array}$ & $\begin{array}{l}1.70 * \\
0.53(0.25)\end{array}$ & $\begin{array}{l}1.61 \\
0.47(0.26)\end{array}$ & $\begin{array}{l}1.61 \\
0.48(0.25)\end{array}$ & $\begin{array}{l}1.66^{*} \\
0.50(0.25)\end{array}$ \\
\hline Married/Partnered (=1) & $\begin{array}{l}1.40 * * * \\
0.33(0.08)\end{array}$ & $\begin{array}{l}1.06 \\
0.06(0.09)\end{array}$ & $\begin{array}{l}1.00 \\
0.00(0.09)\end{array}$ & $\begin{array}{l}1.07 \\
0.06(0.09)\end{array}$ & $\begin{array}{l}1.05 \\
0.05(0.09)\end{array}$ & $\begin{array}{l}1.01 \\
0.01(0.09)\end{array}$ \\
\hline \multicolumn{7}{|l|}{ Socioeconomic Status: } \\
\hline No High School Degree & & $\begin{array}{l}0.96 \\
-0.05(0.10)\end{array}$ & $\begin{array}{l}1.01 \\
0.01(0.10)\end{array}$ & $\begin{array}{l}0.95 \\
-0.05(0.10)\end{array}$ & $\begin{array}{l}1.00 \\
0.00(0.10)\end{array}$ & $\begin{array}{l}1.04 \\
0.04(0.10)\end{array}$ \\
\hline College Degree & & $\begin{array}{l}1.20 * \\
0.18(0.09)\end{array}$ & $\begin{array}{l}1.16 \\
0.15(0.09)\end{array}$ & $\begin{array}{l}1.19 \\
0.17(0.09)\end{array}$ & $\begin{array}{l}1.12 \\
0.11(0.09)\end{array}$ & $\begin{array}{l}1.10 \\
0.09(0.09)\end{array}$ \\
\hline \multicolumn{7}{|l|}{ Income Quartiles: } \\
\hline Lower Middle Quartile & & $\begin{array}{l}1.39 * * \\
0.33(0.11)\end{array}$ & $\begin{array}{l}1.35^{* *} \\
0.30(0.11)\end{array}$ & $\begin{array}{l}1.38 * * \\
0.32(0.11)\end{array}$ & $\begin{array}{l}1.34^{* *} \\
0.29(0.11)\end{array}$ & $\begin{array}{l}1.32 * \\
0.28(0.11)\end{array}$ \\
\hline Upper Middle Quartile & & $\begin{array}{l}1.42 * * \\
0.35(0.12)\end{array}$ & $\begin{array}{l}1.32 * \\
0.27(0.12)\end{array}$ & $\begin{array}{l}1.38 * * \\
0.32(0.12)\end{array}$ & $\begin{array}{l}1.38 * * \\
0.32(0.12)\end{array}$ & $\begin{array}{l}1.29 * \\
0.26(0.12)\end{array}$ \\
\hline Upper Quartile & & $\begin{array}{l}1.50 * * \\
0.41(0.14)\end{array}$ & $\begin{array}{l}1.39 * \\
0.32(0.14)\end{array}$ & $\begin{array}{l}1.46^{* * * *} \\
0.38(0.14)\end{array}$ & $\begin{array}{l}1.48 * * \\
0.39(0.14)\end{array}$ & $\begin{array}{l}1.39 * \\
0.33(0.14)\end{array}$ \\
\hline
\end{tabular}


Wealth Quartiles:

Bottom Quartile (ref.)

Lower Middle Quartile

Upper Middle Quartile

Upper Quartile

Depressive Symptomology

$$
1.23
$$

$0.20(0.11)$

$1.76^{* * *}$

$$
0.16(0.11)
$$$$
1.17
$$

$0.57(0.11)$

$1.93 * * *$

$0.66(0.13)$

$1.67 * * *$

$0.51(0.12)$

$1.83^{* * *}$

$0.61(0.13)$

$0.84 * * *$

$-0.17(0.02)$

\section{Perceived Mastery}

\section{Physical Activity}

Intercept
-2LogLikelihood
F Change
Pseudo-b $^{2}$

0.27
5319.83

Degrees of Freedom

0.029

-0.04
5229.24
$90.59 * * *$
0.053

0.58

5149.67

1.20

$0.18(0.11)$

$1.70 * * *$

$0.53(0.11)$

$1.81 * * *$

0.59 (0.13)

$1.29 * * *$

0.25 (0.04)

$79.87 * * *$

15

16

1.15

$0.14(0.11)$

$1.57 * * *$

$0.45(0.12)$

$1.65 * * *$

$0.50(0.13)$

(n)

$1.11 * * *$

$0.10(0.01)$

$-1.34^{* * *}$

5173.48

$55.76 * * *$

0.074

16
1.10

$0.10(0.11)$

$1.49 * * *$

$0.40(0.12)$

$1.56 * * *$

$0.44(0.13)$

$0.88 * * *$

$-0.13(0.02)$

$1.18 * * *$

$0.16(0.04)$

$1.10 * * *$

0.09 (0.01)

$-1.26^{* *}$

5020.98 $208.26^{* * *}$

0.109

Notes: ${ }^{a}$ Model 2 compared with Model 1; ${ }^{b}$ Models 3-6 compared with Model 2; *p $\leq 0.05 ; * * \mathrm{p} \leq 0.01 ; * * * \mathrm{p} \leq 0.001$ 
Table 4. Unstandardized Regression Coefficient Estimates (and Standard Errors) of Recovery from Mobility Limitation, Depressive Symptomology, Perceived Mastery, and Physical Activity (N=5,922)

\begin{tabular}{|c|c|c|c|c|c|c|c|}
\hline & Model 1 & Model 2 & Model 3 & Model 4 & Model 5 & Model 6 & Model 7 \\
\hline & \multicolumn{4}{|c|}{ Recovery from Mobility Limitation } & $\begin{array}{c}\text { Depressive } \\
\text { Symptomology }\end{array}$ & $\begin{array}{l}\text { Perceived } \\
\text { Mastery }\end{array}$ & $\begin{array}{l}\text { Physical } \\
\text { Activity }\end{array}$ \\
\hline Physical Neighborhood & -0.03 & -0.03 & -0.03 & -0.02 & $0.06 * *$ & $-0.08 * * *$ & -0.07 \\
\hline Disorder & $(0.03)$ & $(0.03)$ & $(0.03)$ & $(0.03)$ & $(0.02)$ & $(0.01)$ & $(0.03)$ \\
\hline Depressive Symptomatology & & $\begin{array}{l}-0.15^{* * *} \\
(0.02)\end{array}$ & $\begin{array}{l}-0.15^{* * *} \\
(0.02)\end{array}$ & $\begin{array}{l}-0.13^{* * *} \\
(0.02)\end{array}$ & & $\begin{array}{l}-0.12 * * * \\
(0.01)\end{array}$ & $\begin{array}{l}-0.26 * * * \\
(0.02)\end{array}$ \\
\hline Perceived Mastery & $\begin{array}{l}0.21^{* * *} \\
(0.04)\end{array}$ & & $\begin{array}{l}0.20^{* * *} \\
(0.04)\end{array}$ & $\begin{array}{l}0.16^{* * *} \\
(0.04)\end{array}$ & $\begin{array}{l}-0.39 * * * \\
(0.02)\end{array}$ & & $\begin{array}{l}0.37 * * * \\
(0.05)\end{array}$ \\
\hline Physical Activity & $\begin{array}{l}0.10 * * * \\
(0.01)\end{array}$ & $\begin{array}{l}0.09 * * * \\
(0.01)\end{array}$ & & $\begin{array}{l}0.09 * * * \\
(0.01)\end{array}$ & $\begin{array}{l}-0.07 * * * \\
(0.01)\end{array}$ & $\begin{array}{l}0.03 * * * \\
(0.00)\end{array}$ & \\
\hline Intercept & -1.99 & -0.37 & -0.52 & -1.26 & 6.67 & 5.36 & 8.25 \\
\hline -2Loglikelihood & 5096.22 & 5042.62 & 5118.11 & 5020.98 & & & \\
\hline Pseudo-R ${ }^{2}$ & 0.098 & 0.103 & 0.083 & 0.109 & & & \\
\hline Degrees of Freedom & 17 & 17 & 17 & 18 & 17 & 17 & 17 \\
\hline$F$ & & & & & 70.02 & 35.92 & 40.32 \\
\hline $\mathrm{R}^{2}$ & & & & & 0.165 & 0.091 & 0.101 \\
\hline
\end{tabular}

Notes: Models 1-4 present unstandardized coefficients (and standard errors) from binary logistic regression; Models 5-7 present unstandardized coefficients (and standard errors) from ordinary least squares (OLS) regression; All models adjusted for demographic characteristics and socioeconomic status; $* \mathrm{p} \leq 0.05 ; * * \mathrm{p} \leq 0.01 ; * * \mathrm{p} \leq 0.001$ 
Figure 1. Conceptual model illustrating potential mediating pathways between neighborhood disorder and recovery from mobility limitation.

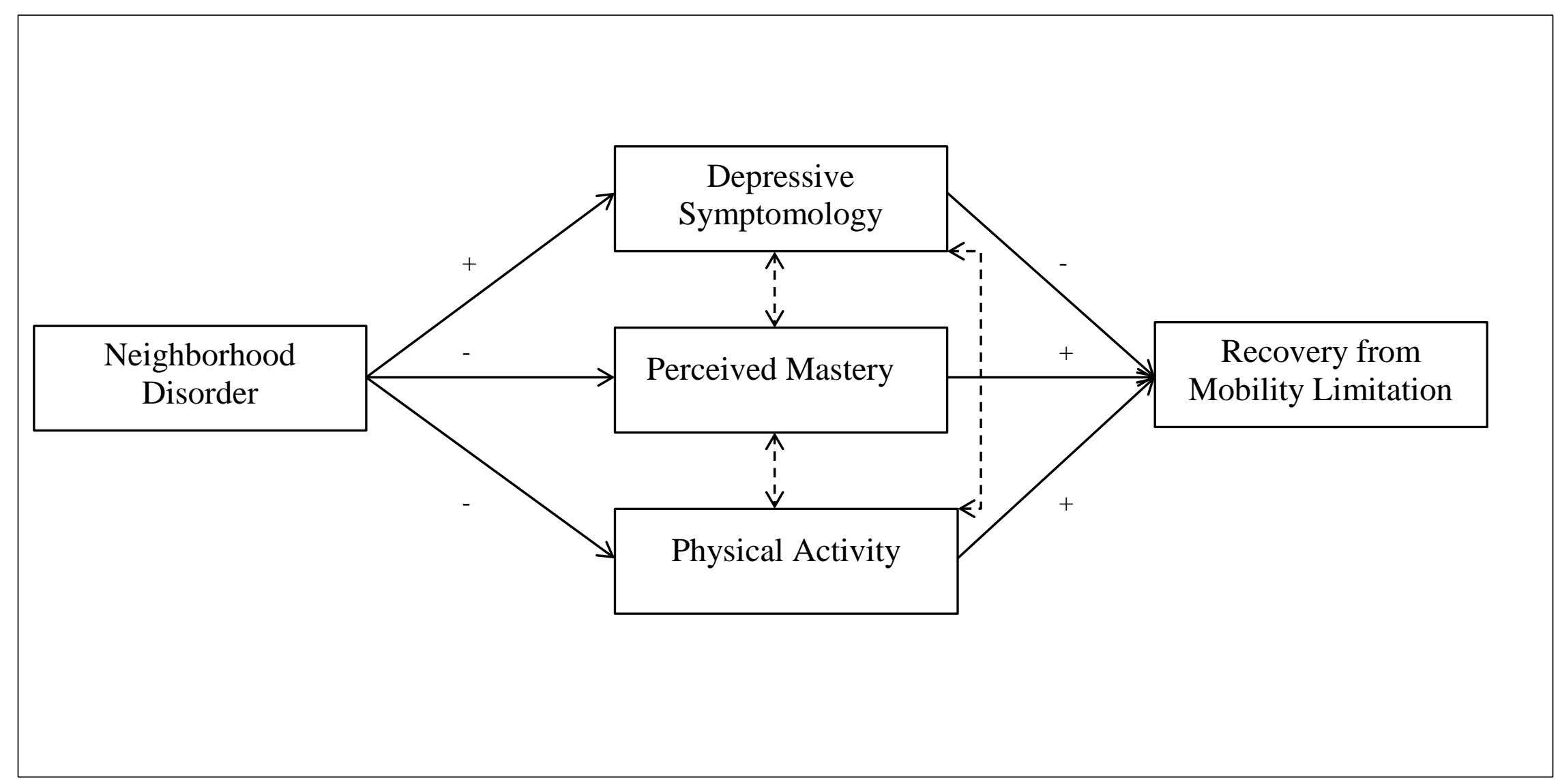

